\title{
Chest Pain in a Young Basketball Player
}

\author{
Catherine Y. Campbell, MD, ' Janet D. Record, MD, 'Aravindan Kolandaivelu, MD, ${ }^{7}$ \\ Roy C. Ziegelstein, $\mathrm{MD}^{1}$ \\ 'Johns Hopkins University School of Medicine, Baltimore, MD, USA.
}

\begin{abstract}
A 32-year-old man was elbowed in the chest while fighting for a rebound in a recreational basketball game. He fell to the ground and his chest ached from the blow. Four days later he developed more severe chest pressure with dyspnea and came to the hospital. His chest wall was tender and his pulse slow, but the remainder of his physical examination was normal. Electrocardiogram showed sinus bradycardia, first-degree atrioventricular (AV) block, and occasional isorhythmic AV dissociation, but no ischemic ST-T changes. Cardiac troponin I rose to $1.74 \mathrm{ng} / \mathrm{mL}$ (normal $<0.50$ ). The patient therefore underwent coronary angiography, showing spiral dissection of the right coronary artery with extensive thrombus filling the distal portion of the vessel. Stenting was unsuccessful in restoring flow. This case highlights the potential dangers of blunt chest trauma in recreational sports and shows how angiography can distinguish myocardial contusion from coronary artery dissection.
\end{abstract}

KEY WORDS: coronary artery dissection; coronary artery injury; blunt chest trauma; blunt cardiac trauma.

DOI: $10.1111 /$ j. 1525-1497.2006.00400.x

J GEN INTERN MED 2006; 21:C7-C10.

A 32-year-old African-American recreational basketball player presented to the emergency department with chest pain. Four days earlier, he was playing basketball with friends and was elbowed in the chest while jumping for a rebound. The impact threw him to the ground. Although he felt that the blow "knocked the wind out of me," he got up a short time later and continued the game. Despite residual chest ache, his exercise tolerance remained excellent. On the day of admission, he was playing basketball again when he developed severe substernal chest pressure associated with lightheadedness, dizziness, and shortness of breath. Another player called for an ambulance, but his symptoms improved by the time emergency personnel arrived, and he declined transport to the hospital. He then walked home, but his chest pressure and lightheadedness recurred and he drove himself to the emergency room. En route, he developed shortness of breath, left arm tingling, and nausea.

Physical exam revealed a comfortable appearing, physically fit African-American man, with a temperature of $97.3^{\circ} \mathrm{F}$, pulse 51 beats per minute, blood pressure 107/75 mm Hg, and oxygen saturation $99 \%$ while breathing room air. He was 67 inches tall and weighed 156 pounds. Cardiovascular exam showed normal jugular venous pressure and a regular rhythm without murmurs or pericardial rub. His lungs were clear. The anterior chest wall was tender. Musculoskeletal exam revealed

The authors have no conflicts of interest to report

Address correspondence and requests for reprints to Dr. Ziegelstein: Department of Medicine, Johns Hopkins Bayview Medical Center, 4940 Eastern Avenue, Baltimore, MD 21224 (e-mail: rziegel@jhmi.edu). normal stature, normal joints without laxity, and no arachnodactyly or chest wall deformity. Skin exam showed normal elasticity.

The initial electrocardiogram showed isorhythmic atrioventricular (AV) dissociation; subsequent electrocardiograms showed sinus bradycardia and borderline first degree AV block (Fig. 1). Cardiac enzymes were initially normal; however, 9 hours later, cardiac troponin I (cTnI) was elevated at $1.74 \mathrm{ng}$ / $\mathrm{mL}$, the creatine phosphokinase (CPK) was $418 \mathrm{U} / \mathrm{L}$, and CPK$\mathrm{MB}$ was $33 \mathrm{ng} / \mathrm{mL}$ (Table 1). A transthoracic echocardiogram showed mild concentric left ventricular hypertrophy and normal left and right ventricular function; there was no pericardial effusion. Therapy with aspirin, intravenous heparin, and eptifibatide was begun, and the patient was taken to the cardiac catheterization laboratory because of a suspicion of ongoing ischemia involving the blood supply to the sinus and AV nodes. Coronary angiography showed large coronary vessels with extensive thrombus in the mid-right coronary artery (RCA) and spiral dissection into all major epicardial branches of the vessel (Fig. 2). Overlapping stents were placed proximal to the origin of the posterior descending artery, but no significant flow was restored. Coronary artery bypass graft surgery was deemed impossible because the dissection had propagated to the distal portion of the RCA. An ascending aortic angiogram showed no aortic dissection. Following stent placement, clopidogrel was added to aspirin, and heparin and eptifibatide were discontinued.

The patient was monitored in the cardiac intensive care unit and an angiotensin converting enzyme (ACE) inhibitor was added. Beta-blockers were avoided because of persistent bradycardia. The cTnI peaked at $5 \mathrm{ng} / \mathrm{mL}$ (Table 1). Repeat transthoracic echocardiogram showed normal left and right ventricular function. The patient recovered uneventfully and was discharged after 3 days. Unfortunately, he did not return for his follow-up appointment in cardiology clinic and his contact information was no longer accurate when attempts were made to try to reach him.

\section{DISCUSSION}

Blunt chest trauma is a well-recognized cause of injury to the heart. ${ }^{1}$ A variety of cardiac injuries may result, including myocardial contusion, intramyocardial hematoma, ventricular rupture, valve injury, pericardial injury with or without tamponade, pericarditis, and coronary artery injury, which may lead to myocardial ischemia or infarction (Table 2). ${ }^{2}$

Manuscript received August 2, 2005

Initial editorial decision September 7, 2005

Final acceptance November 20, 2005 


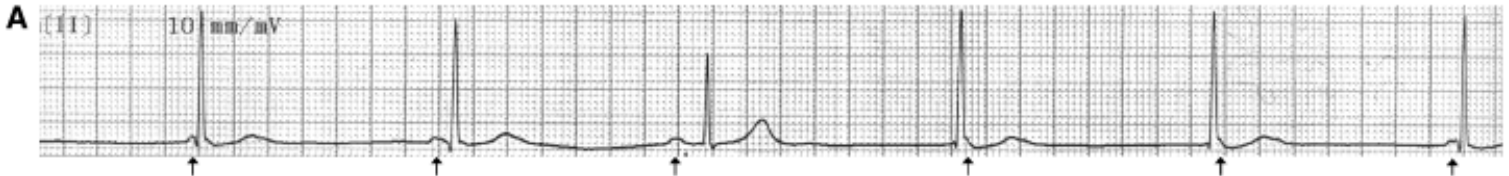

B

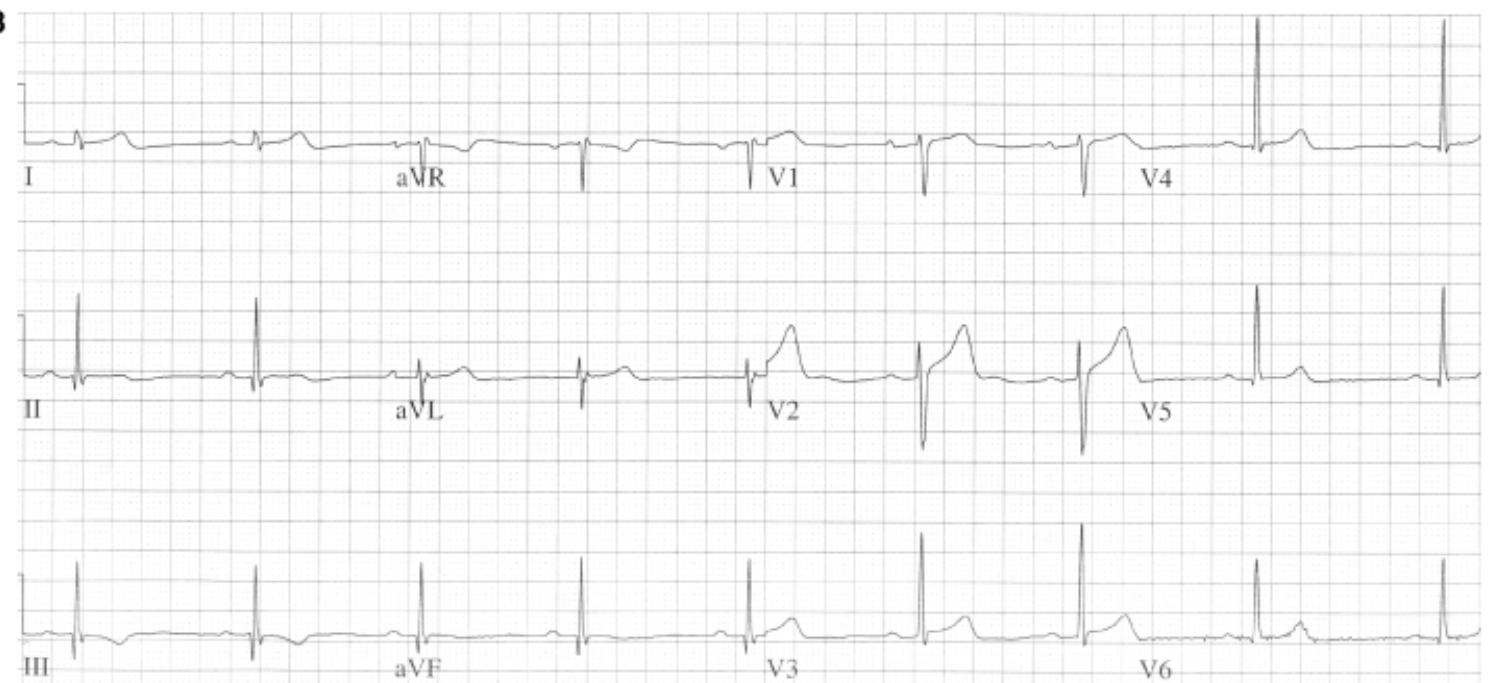

FIGURE 1 Isorhythmic atrioventricular (AV) dissociation. (A) The electrocardiogram at presentation showed isorhythmic AV dissociation. The P wave (indicated by arrows) demonstrates no consistent relationship with the QRS complex. (B) The electrocardiogram during recovery 1 day later showed sinus bradycardia and borderline first degree AV block.

Myocardial contusion is the most common cardiac complication of blunt chest trauma. The incidence of myocardial contusion in autopsy series of motor vehicle accident victims ranges from about $16 \%$ in unselected patients to $76 \%$ in cases of more severe trauma. ${ }^{2}$ However, coronary artery injury resulting from blunt chest trauma is unusual. Case series and autopsy reviews suggest a $1 \%$ to $2 \%$ incidence of coronary artery injury following significant blunt chest trauma. ${ }^{2}$ Among cases of coronary artery injury, dissection is a particularly rare complication. Motor vehicle accidents are the most common cause of traumatic coronary artery dissection. ${ }^{2,3}$ Injury to the coronary arteries following milder trauma due to sporting accidents is much less common, but cases of sports-related traumatic coronary artery dissection have been reported following waterskiing ${ }^{4}$ and bicycle ${ }^{5}$ accidents and after collisions during basketball, ${ }^{6}$ soccer, ${ }^{7}$ and rugby games. ${ }^{8}$ Blunt chest trauma may also cause injury to other vessels, including the thoracic aorta and the subclavian and internal mammary arteries. However, injury to these vessels usually requires much more severe trauma, such as a high-speed motor vehicle collision, and is typically accompanied by other injuries such as frac-

Table 1. Cardiac Enzyme Measurements

\begin{tabular}{llllllll}
\hline \hline Day & \multicolumn{9}{c}{1} & & \multicolumn{2}{c}{2} \\
\cline { 2 - 4 } \cline { 8 - 9 } Time (h) & $\mathbf{0 1 : 3 0}$ & $\mathbf{1 0 : 2 0}$ & $\mathbf{1 7 : 1 0}$ & $\mathbf{2 1 : 1 5}$ & & $\mathbf{0 6 : 3 0}$ & $\mathbf{1 2 : 3 0}$ \\
\hline CPK & 287 & 418 & 595 & 560 & & 455 & 408 \\
CPK-MB & 4 & 33 & 46 & 30 & & 31 & 26 \\
cTnI & 0.09 & 1.74 & 5.00 & 3.65 & & 2.84 & \\
\hline
\end{tabular}

Normal values: CPK 24-195 IU/L; CPK-MB 0 to $7 \mathrm{ng} / \mathrm{mL}$; cardiac troponin I (cTnI) 0.00 to $0.50 \mathrm{ng} / \mathrm{mL}$.

CPK, creatine phosphokinase. tures, dislocations, brachial plexus injury, and pulmonary contusions. ${ }^{9,10}$ Because of its anterior location, the left anterior descending artery is the most frequently injured epicardial vessel, followed by the right coronary and circumflex arteries. ${ }^{2-4,11}$ The right coronary artery may be more prone to injury when blunt chest trauma occurs during ventricular systole, a time when this vessel lies in a more vulnerable anterior position. ${ }^{12}$ The mechanism of dissection involves deceleration and shearing forces, causing an intimal tear or subintimal hemorrhage, which proceeds to dissection and luminal thrombus formation, often causing myocardial infarction. ${ }^{2,3,12}$ Significant delay between blunt trauma and dissection is unusual, but can result from gradual enlargement of the false lumen and progressive impingement into the true lumen. ${ }^{4,13-15}$ Occlusion of the true lumen results from enlargement of the false lumen and from thrombosis induced by intimal injury. If sufficient occlusion of the true lumen occurs, myocardial infarction results.

When managing patients who report recent blunt chest trauma and present with chest pain accompanied by rising markers of myocardial injury, a transthoracic echocardiogram helps to narrow the differential diagnosis by assessing for valvular abnormalities, pericardial effusion, and the presence and extent of wall motion abnormalities. In cases where transthoracic echocardiography may be limited by consequences of other injuries sustained in chest trauma, including hemothorax, pneumothorax, or rib fractures, transesophageal echocardiography has been used. However, echocardiography is insensitive for myocardial contusions of only mild to moderate severity. ${ }^{2}$ In the present case, no valvular or pericardial abnormalities were seen using transthoracic echocardiography; thus the most likely explanation for the rising levels of cardiac enzymes was either myocardial contusion or coronary artery 

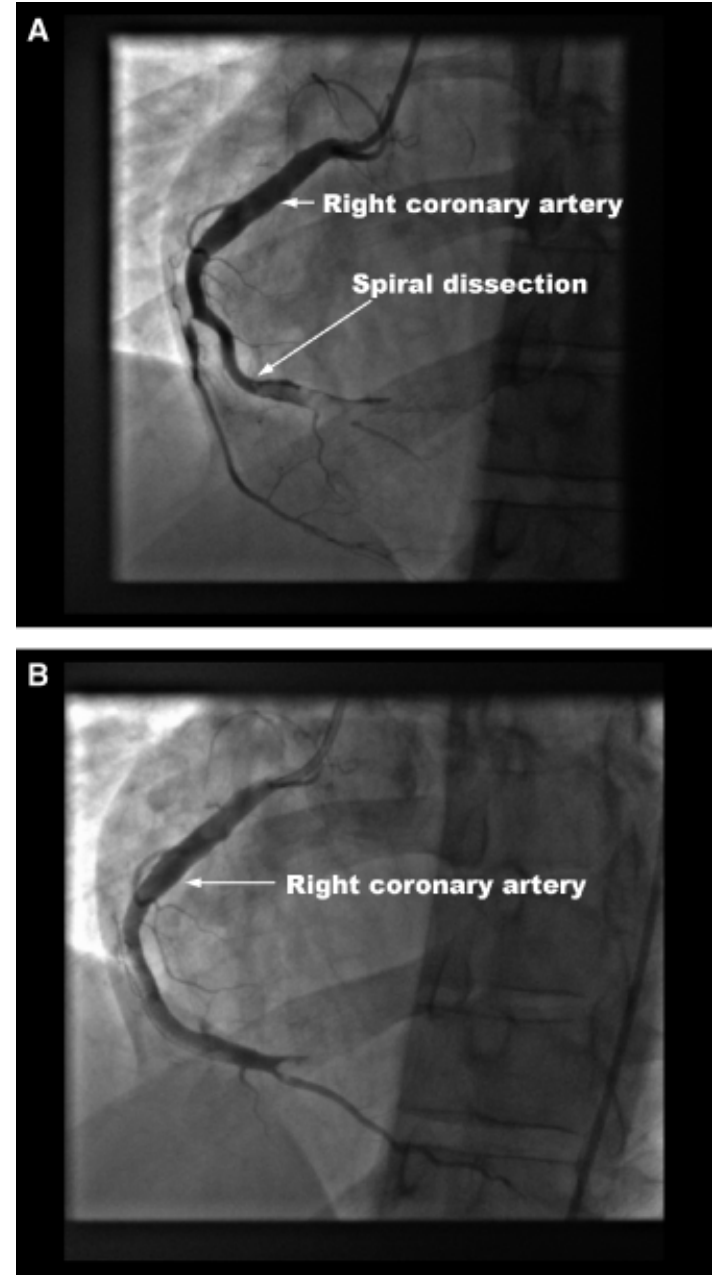

FIGURE 2 Right coronary artery dissection coronary angiography (left anterior oblique projection) showing extensive thrombus (arrow) in the distal right coronary artery and spiral dissection (arrowheads) into all major epicardial branches of the vessel. (A) Preintervention. (B) Postintervention, demonstrating failure to reperfuse distal right coronary artery.

occlusion with myocardial infarction. That distinction was important to make, as intervention may be possible in cases of coronary artery occlusion. Thus the next step was emergent cardiac catheterization. In cases of myocardial contusion, angiography reveals coronary arteries without thrombus. Catheterization in this case revealed an intimal flap and spiral dissection of the RCA with thrombus filling the vessel (Fig. 2).

Noninvasive coronary artery imaging using multislice computed tomography (CT angiography) is emerging as an important diagnostic tool to evaluate coronary arteries and soon may be more commonly used in cases of possible dissection. This modality offers several advantages over conventional angiography. Computed tomography angiography is noninvasive, rapid, and is able to image both the vessel wall and lumen; it also readily identifies anomalous coronary anatomy. In one report of a patient with spontaneous coronary artery dissection, CT angiography provided better depiction of the extent of an intramural hematoma than conventional coronary angiography. ${ }^{16}$ Although CT angiography appears to be an effective method for diagnosing coronary dissection and formulating a treatment plan, it does not represent a mode of treatment itself
Table 2. Differential Diagnosis of Cardiac Injuries After Blunt Chest Trauma

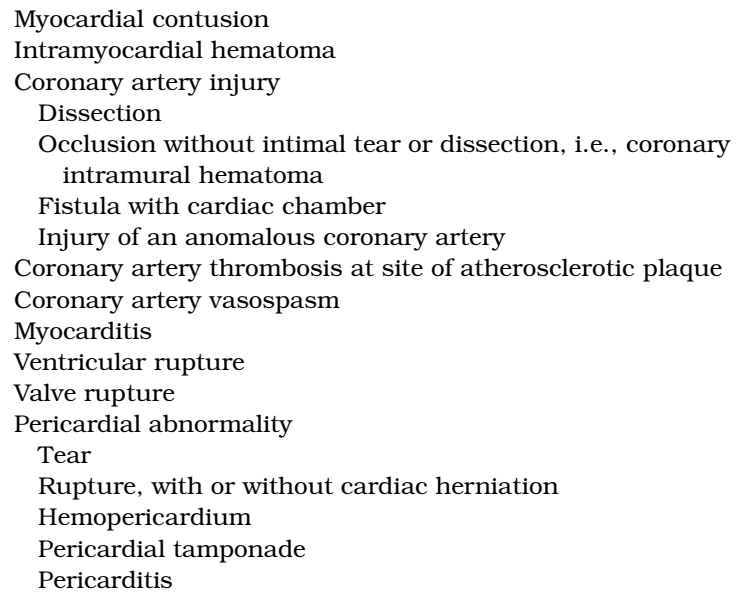

and should not delay emergent conventional angiography which allows simultaneous percutaneous coronary intervention. ${ }^{17}$

The optimal management of traumatic coronary artery dissection is uncertain. ${ }^{15}$ No trials directly comparing various treatment options have been performed, most likely because of the rare incidence of the condition. Management options include medical therapy with anticoagulation and antiplatelet agents; catheterization with angioplasty, with or without stenting; and coronary artery bypass graft surgery. ${ }^{15}$ In some cases, thrombolytic therapy has been given early in the course, before coronary angiography, because of electrocardiographic evidence of coronary artery occlusion and transmural ischemia in the setting of chest pain. Thrombolytic therapy in cases of myocardial infarction because of coronary dissection resulted in good outcomes in 2 studies, ${ }^{8,15}$ but led to extension of the dissection in another. ${ }^{17}$ Any anticoagulant, antiplatelet, or thrombolytic therapy has the potential to propagate the dissection by promoting hemorrhage into the false lumen. In cases where there is a high suspicion of coronary artery dissection, as in young patients with few risk factors for atherosclerosis and a recent history of chest trauma, angiography should be performed early and thrombolytics avoided until dissection can be excluded. ${ }^{15,18}$ Treatment of coronary dissection should be guided by the patient's clinical condition and the extent and location of the dissection. Although the initial management of patients with this condition is typically directed by, or done in consultation with, a cardiologist, the General Internist plays a critical role often in the initial recognition of this condition and in its medical management. Medical management alone is appropriate for hemodynamically stable patients with normal left ventricular systolic function and for those whose coronary anatomy prohibits percutaneous or surgical revascularization. Medical management should include continuous monitoring for arrhythmias for a minimum of 24 hours, use of ACE inhibitors for favorable effects on left ventricular remodeling, and $\beta$-blockers as tolerated.

Prognosis in cases of coronary dissection where patients survive to hospital admission varies according to the amount of ischemic myocardium and the residual left ventricular systolic function. In our patient's case, left ventricular function remained normal and cardiac enzyme elevation was fairly minor, 
perhaps due to collateral flow from other vascular territories in a patient who had no evidence of disease in other vascular beds. Many patients with traumatic coronary artery dissection due to motor vehicle accidents do not survive to hospital admission. ${ }^{2}$ Some cases of sudden death in sports-related blunt chest trauma that are attributed to commotio cordis (ventricular arrhythmia induced by blunt chest trauma) may in fact represent cases of coronary dissection and myocardial infarction. ${ }^{8}$ When patients survive to hospital admission, prognosis appears to be favorable. In 1 small series of coronary injuries from blunt chest trauma, follow-up angiography showed complete healing of coronary artery injury by 6 months, which is similar to outcomes in cases of intimal injury caused by trauma during cardiac catheterization. ${ }^{11}$ Development of a coronary artery aneurysm at the site of dissection can be a late complication, illustrating the importance of repeat coronary imaging months to years after initial presentation, especially in cases that were managed medically. Re-evaluation of left ventricular systolic function is also indicated. ${ }^{11}$

The patient's distinctive admission electrocardiogram deserves further discussion. The electrocardiogram showed marked bradycardia and isorhythmic AV dissociation. Isorhythmic AV dissociation is a specific form of conduction block in which the atria and ventricles are electrically dissociated (i.e., AV dissociation), and in which the atrial rate is virtually identical to the junctional or ventricular escape rate. Bradycardia and AV dissociation can be normal findings in a wellconditioned athlete like the patient presented here. ${ }^{19}$ Given this, coupled with the initially nondiagnostic cardiac enzyme levels and the absence of acute ischemic changes on the electrocardiogram, it would not have been surprising if this patient's evaluation had been delayed. In addition, this young man appeared comfortable and did not complain of much pain, which initially led him to refuse ambulance transport to the hospital. However, the bradycardia and AV dissociation, mildly elevated markers of myocardial damage, and classic ischemic symptoms raised concern for acute myocardial ischemia or infarction, possibly affecting the inferior wall of the left ventricle and the blood supply of the AV node. This concern led to prompt evaluation and management in the emergency department and to early consultation with cardiology.

This case demonstrates an unusual occurrence of delayed right coronary artery dissection following blunt chest trauma during a basketball game. Coronary artery dissection should be included in the differential diagnosis of patients presenting with chest pain following blunt chest trauma. This case highlights the importance of maintaining a high index of suspicion for coronary artery injury in an otherwise healthy individual presenting after blunt chest trauma with chest pain and clinical and laboratory evidence of myocardial damage. Coronary angiography should be considered early in such cases to distinguish between myocardial contusion and potentially treatable coronary occlusion.
Drs. W. Lowell Maughan and David R. Thiemann performed cardiac catheterization and percutaneous coronary intervention.

\section{REFERENCES}

1. Parmley LF, Manion WC, Mattingly TW. Nonpenetrating traumatic injury of the heart. Circulation. 1958;18:371-96.

2. Salehian O, Teoh K, Mulji A. Blunt and penetrating cardiac trauma: a review. Can J Cardiol. 2003;19:1054-9.

3. Vlay SC, Blumenthal DS, Shoback D, Fehir K, Bulkley BH. Delayed acute myocardial-infarction after blunt chest trauma in a young woman. Am Heart J. 1980; 100:907-16.

4. Greenberg J, Salinger M, Weschler F, Edelman B, Williams R. Circumflex coronary artery dissection following waterskiing. Chest. 1998; 113:1138-40.

5. Gustavsson CG, Albrechtsson U, Forslind K, Stahl E, White T. A case of right coronary artery occlusion, caused by blunt chest trauma and treated with acute coronary artery bypass surgery. Eur Heart J. 1992;13: 133-6.

6. Moore JE. Acute apical myocardial infarction after blunt chest trauma incurred during a basketball game. J Am Board Fam Pract. 2001;14: 219-22.

7. Dahle TG, Berger A, Tuna N, Das G. Coronary artery stenting for acute myocardial infarction secondary to mild, blunt chest trauma in a soccer player. J Invasive Cardiol. 2005;17:163-4.

8. Vasudevan AR, Kabinoff GS, Keltz TN, Gitler Blunt chest trauma producing acute myocardial infarction in a rugby player. Lancet. 2003; 362:370.

9. Kwon OY, Chung SP, Yoo IS, Song CJ, Kim IB, Kim Sw. Delayed presentation of internal mammary artery rupture after blunt chest trauma: characteristic CT and plain X ray findings. Emerg Med J. 2005;22: 664-5.

10. Madoff DC, Brathwaite CE, Manzione JV, et al. Coexistent rupture of the proximal right subclavian and internal mammary arteries after blunt chest trauma. J Trauma. 2000;48:521-4.

11. Ginzburg E, Dygert J, Parra-Davila E, Lynn M, Almeida J, Mayor M. Coronary artery stenting for occlusive dissection after blunt chest trauma. J Trauma. 1998;45:157-61.

12. Oliva PB, Hilgenberg A, McElroy D. Obstruction of the proximal right coronary artery with acute inferior infarction due to blunt chest trauma. Ann Intern Med. 1979;91:205-7.

13. Boland J, Limet $\mathbf{R}$, Trotteur G, Legrand V, Kulbertus $\mathbf{H}$. Left main coronary dissection after mild chest trauma - favorable evolution with fibrinolytic and surgical therapies. Chest. 1988;93:213-4.

14. Hazeleger R, van der Wieken $\mathbf{R}$, Slagboom T, Landsaat P. Coronary dissection and occlusion due to sports injury. Circulation. 2001;103: 1174-5.

15. Kerwin TC, Ruggie N, Klein LW. Spontaneous coronary artery dissection following low-intensity blunt chest trauma: a case report and review of current treatment options. J Invasive Cardiol. 2002;14: 679-81.

16. Manghat NE, Morgan-Hughes GH, Roobottom CA. Spontaneous coronary artery dissection: appearance and follow-up on multi-detector row CT coronary angiography. Clin Radiol. 2005;60:1120-5.

17. Schoenhagen P, Stillman AE, Halliburton SS, White RD. CT of the heart: principles, advances, clinical uses. Cleveland Clin J Med. 2005; 72:127-38.

18. Buys EM, Suttorp MJ, Morshuis WJ, Plokker HW. Extension of a spontaneous coronary artery dissection due to thrombolytic therapy. Cathet Cardiovasc Diagn. 1994;33:157-60.

19. Viitasalo MT, Kala R, Eisalo A. Ambulatory electrocardiographic recording in endurance athletes. Br Heart J. 1982;47:213-20. 